\title{
Interaction of femtosecond laser radiation with carbon materials: exfoliation of graphene structures and synthesis of low-dimensional carbon structures
}

\author{
D. Abramov, S. Arakelian, D. Kochuev, S. Makov, V. Prokoshev, K. Khorkov \\ Vladimir State University, Vladimir, Russia \\ awraam@mail.ru, arak@vlsu.ru,_b_@mail.ru,stephan.makov@gmail.com, \\ prokoshev_vg@vlsu.ru, freeod@mail.ru
}

PACS 81.05.-t, 42.62.Cf

DOI 10.17586/2220-8054-2016-7-1-220-225

Carbon is represented in modern nanomaterials by a large variety of modifications. Various methods and technologies have been developed to create these various forms. Methods utilizing laser irradiation constitute a large portion of these techniques. The action of laser pulses upon graphite may result in the exfoliation of graphene layers. This paper presents the results of implementing method of laser-induced cleavage of graphite in liquid nitrogen using femtosecond laser radiation pulses. The process of obtaining graphene from the laser processing of graphite is accompanied by the formation of various types of low-dimensional carbon structures.

Keywords: graphene, carbon nanorods, laser synthesis, femtosecond laser.

Received: 20 November 2015

\section{Introduction}

Carbon is the basis for many of nanostructures and nanomaterials. Carbon nanotubes, fullerenes and graphene are the most well-known carbon nanostructures, however, this is not an exhaustive list of such materials. Graphene has attracted the attention of many scientists and industrial researchers due to its unique physical and chemical properties. However, the lack of a universal and inexpensive method for its synthesis hampers investigation of its properties and wide application of this promising material. Currently, numerous methods are available for the fabrication of graphene [1]. Among them are such basic techniques as micromechanical exfoliation of graphite, liquid phase exfoliation of graphite, graphite oxidation, chemical vapor deposition method, electric arc graphene production, thermal decomposition of silicon carbide, epitaxial growth of graphene on a metal surface and others. All these methods have their particular advantages and disadvantages. Therefore, research towards the development of methods for graphene fabrication has been extensively carried out.

The liquid phase exfoliation of graphite is a logical development of the classical method of micromechanical graphene fabrication [2]. The use of special chemical and technical conditions makes this method more efficient. Liquid surfactants weaken the bonds between the graphite layers. Ultrasonic treatment or centrifugation are then used to separate the graphene sheets. The development of the liquid-phase graphite exfoliation method has occurred with the aim of finding new solvents which may offer novel approaches for the separation of graphene sheets, which can provide increases in the synthetic yields and purity of the obtained graphene. We propose to use the liquid nitrogen and laser-induced exfoliation for these purposes.

The interaction of femtosecond laser radiation with materials is often accompanied by the formation of micro- and nanostructures on the irradiated surface [3]. The formation of nanorods or nanotips arrays in the case of structuring on the surface of carbon targets is a 
distinct sub-interest. Such structures have potential for use in field emission displays or for hydrogen storage [4]. We have registered the formation of arrays of nanorods on the stage preceding the separation of graphene. These results are also presented in this paper.

\section{Methods}

Laser-induced liquid-phase exfoliation of graphite has been realized using a femtosecond laser radiation pulse. During this exfoliation the aim is to heat only the surface layer of a graphite target with the minimum possible thickness required to separate the surface from the graphene sheet layers underneath. Lasers with the least possible pulse duration of radiation can provide such conditions. Currently, femtosecond-pulse lasers are best suited for this purpose. Two femtosecond laser systems have been used in experiments for the fabrication of graphene. They are ytterbium industrial setup TETA-10 and experimental Ti:sapphire femtosecond laser system.

The irradiation parameters of TETA-10 were as follows: laser radiation wavelength $\lambda=1029 \mathrm{~nm}$, pulse with $\tau=300 \mathrm{fs}$, energy per pulse $\varepsilon=0.15 \mathrm{~mJ}$ and pulse repetition frequency $f=10 \mathrm{kHz}$. This laser system was used in the first series of experiments. The reason for beginning this research with the TETA-10 laser system was because it is widely available for commercial industrial use. The energy characteristics of Ti:sapphire laser are larger and the radiation pulse is shorter than similar parameters for the ytterbium laser. These facts were the basis for theorizing about the possibility of increasing the speed of the graphene generation process when using such laser. Therefore, this latter system was used in the second series of experiments, which was performed to verify of the proposed hypothesis. The irradiation parameters for the $\mathrm{Ti}$ : sapphire laser system were as follows: laser radiation wavelength $\lambda=800 \mathrm{~nm}$, pulse with $\tau=50 \mathrm{fs}$, energy per pulse $f=1 \mathrm{~mJ}$ and pulse repetition frequency $f=1 \mathrm{kHz}$. The laser beam was focused on the target surface in a spot with a diameter of $100 \mu \mathrm{m}$ in both series of experiments. The laser beam was moved along the target surface at a speed of $0.01 \mathrm{~m} / \mathrm{s}$ to $1 \mathrm{~m} / \mathrm{s}$.

Samples of the highly oriented pyrolytic graphite (HOPG) and glassy carbon were used as sources for obtaining the graphene. Laser processing of carbon targets was carried out in liquid nitrogen. The depth of a liquid nitrogen layer above the target surface was $\sim 10 \mathrm{~mm}$. The liquid nitrogen molecules easily penetrate into spaces between graphite layers. Once in between the graphene layers, the cryogenic liquid then undergoes evaporation during laser exposure. The gas phase expands, thus separating sheets of graphene. Successful application of liquid nitrogen in the liquid phase exfoliation of graphite was displayed in [5]. The authors of this paper used a Q-switched Nd:YAG nanosecond laser system. The process for obtaining graphene material lasted 20 minutes. Using femtosecond laser radiation allowed us to significantly enhance the rate of grapheme fabrication while maintaining comparable characteristics.

Moreover, liquid nitrogen also serves another function; it provides the rapid cooling of the irradiated target surface and attenuates the results of laser irradiation. Thus, potentially destructive laser processing side-reactions are minimized. We have successfully applied this approach to study the interaction of femtosecond laser radiation pulses on metals [6]. This has also shown good results in experiments on the synthesis of graphene and low-dimensional carbon structures.

The structures formed under femtosecond laser irradiation in liquid nitrogen were investigated by analyzing the images obtained using a scanning electron microscope (SEM) Quanta $2003 \mathrm{D}$. 


\section{Experimental results}

Multi-layer graphene was obtained in both series of experiments. Exposure to a single laser pulse did not exfoliate the graphene sheets. Doing so only modified or destroyed the target surface (depending on the laser radiation intensity). Exfoliation graphene takes place under the influence of four laser pulses. Examples of obtained graphene structures are shown in Fig. 1. Exposure of fifth pulse femtosecond radiation leads to the destruction of obtained nanostructures. Irradiation with a required number of pulses has been provided by appropriate overlap of areas of laser action. The required overlap (75\%) corresponds to the velocity for the movement of the laser beam along the target surface $0.25 \mathrm{~m} / \mathrm{s}$ at using of a ytterbium laser and $0.025 \mathrm{~m} / \mathrm{s}$ in the case of treatment of graphite by of Ti : sapphire laser irradiation.

Graphene ribbons with a width up to $50 \mu \mathrm{m}$ and a length of more than $150 \mu \mathrm{m}$ and also an arbitrary shape graphene plates with a characteristic size up to $150 \mu \mathrm{m}$ were registered after treatment of HOPG by ytterbium laser irradiation. The obtained graphene ribbons have folds but also have sufficiently extensive smooth surface portions (an area up to $2000 \mu \mathrm{m}^{2}$ ).

The graphene material fabricated with the use of Ti : sapphire laser consisted mainly of structures with linear dimensions on the order of a few micrometers. Typically cleaved sheets have numerous folds, although the individual sheets were characterized by a smooth surface and small number of folds. Some registered graphene structures ranged in size from 10 to $30 \mu \mathrm{m}$.

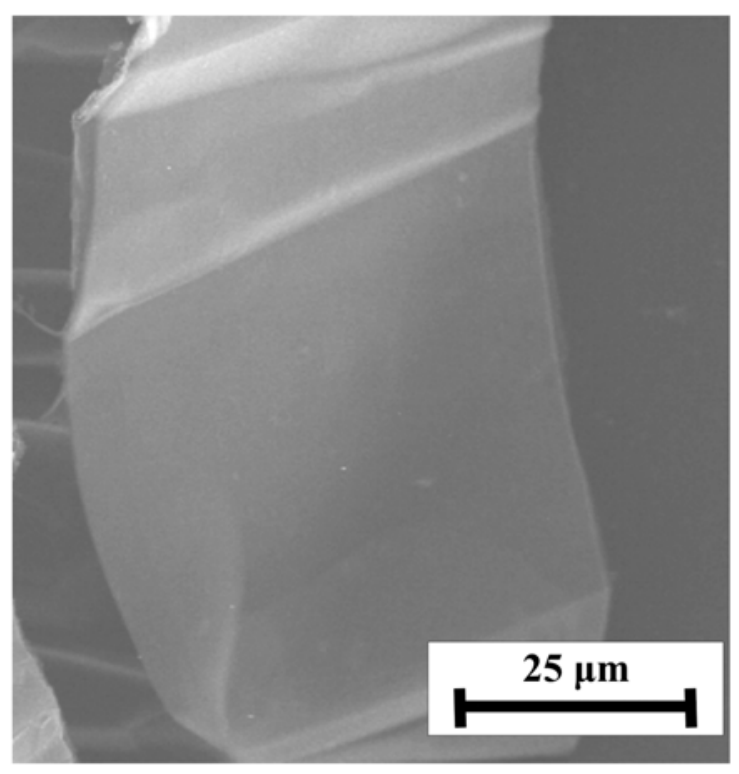

(a)

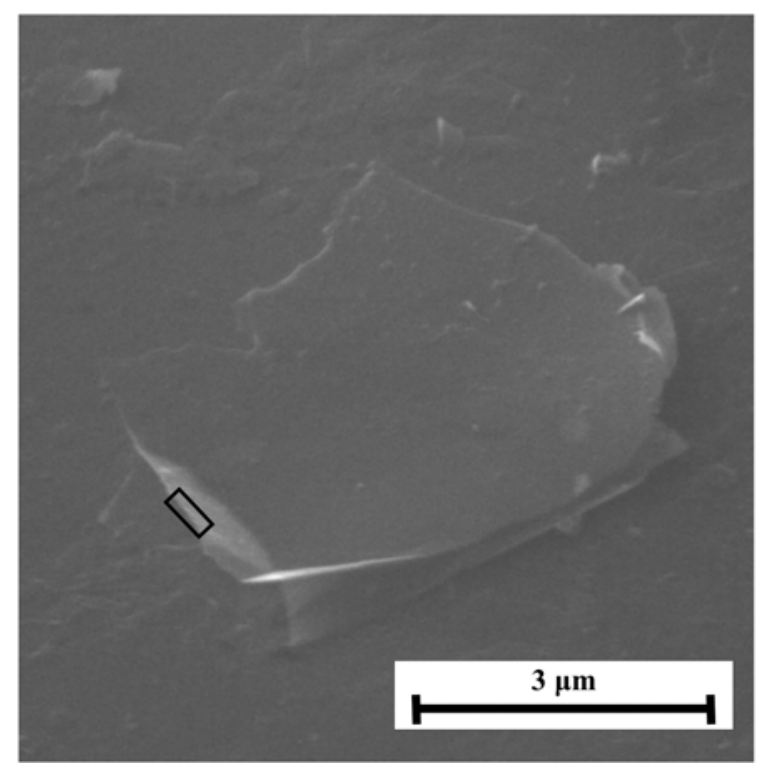

(b)

FIG. 1. Graphene structures on the HOPG surface: a - processing of graphite by radiation of ytterbium laser; $\mathrm{b}$ - processing of graphite by radiation of $\mathrm{Ti}$ : sapphire laser

Analysis of the SEM images of end faces of obtained structures allowed us to estimate their transverse dimensions. Graphene sheets with a thickness of $10 \mathrm{~nm}$ were detected (Fig. 2). It should be noted that traditional methods of micromechanical or liquid-phase exfoliation of graphite basically provides fabrication of a multilayer graphene sheets with a thickness of about $16 \mathrm{~nm}$ (according to [1]).

Laser treatment of surface of a glassy carbon samples in liquid nitrogen resulted in the fabrication of graphene in the form of crumpled sheets with characteristic structural sizes ranging from 1 to $2 \mu \mathrm{m}$ and sheet thickness of about $20 \mathrm{~nm}$. 


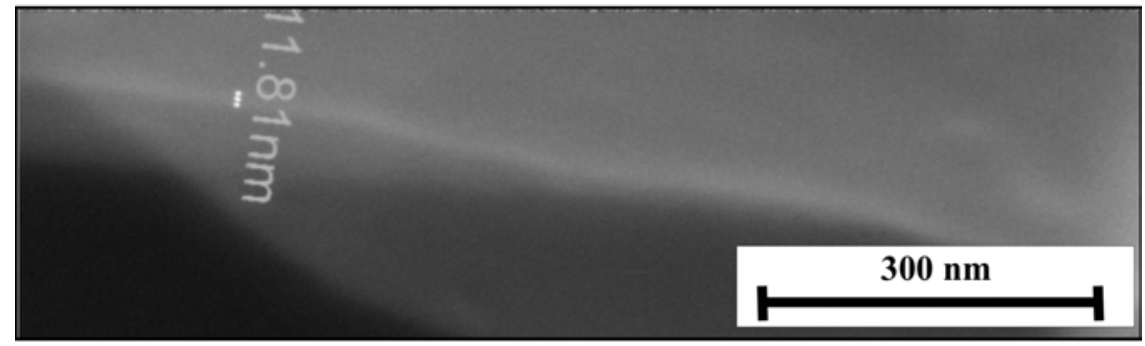

FIG. 2. Measuring the thickness of graphene structures. SEM image corresponds to an area that is highlighted by black rectangle in Fig. 1b

Carbon nanostructures in the form of vertical nanorods were formed on the surface of a graphite target when the number of pulses of laser radiation was less than that necessary for graphene sheet exfoliation. Areas with a random and regular arrangement of the nanorods are registered on the laser modified surface. In the latter case, nanorods are arranged in rows (Fig. 3). Here, nanorods have a diameter of $\sim 100 \mathrm{~nm}$, with a distance between rows (period of a total structure) of $\sim 100 \mathrm{~nm}$. The distance between the rods in a row is $\sim 100 \mathrm{~nm}$. The periodicity of the rows is 8 times smaller than the wavelength of the light used in the laser irradiation experiment. This ratio corresponds to the concept about the discrete changes in the periodicity of the regular structures of the relief, which is formed via the interaction of laser radiation with condensed media. This concept exists in the framework of the universal polariton model (see, for example, the review [7]). Moreover, the spatial changes must be multiples of the main period, which is determined by the wavelength of incident radiation.

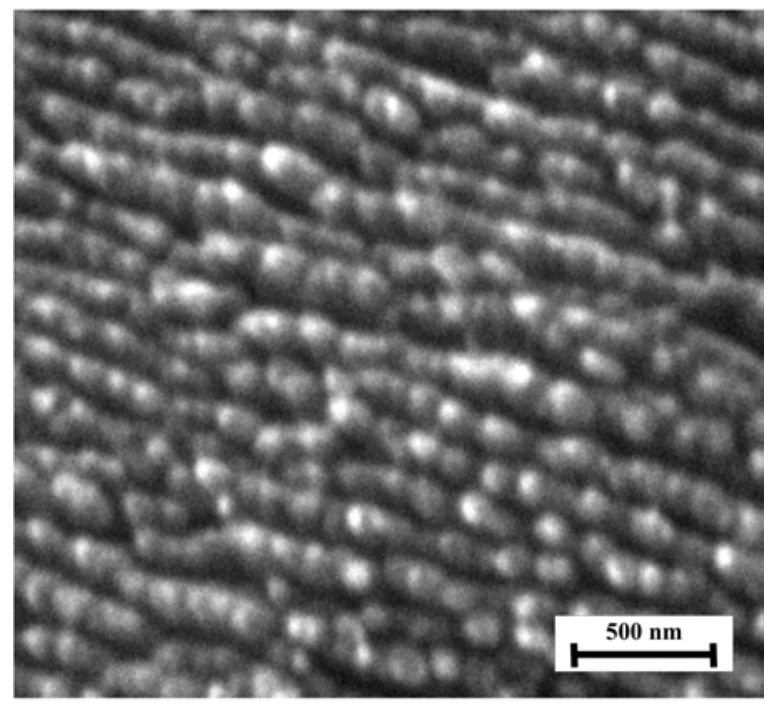

(a)

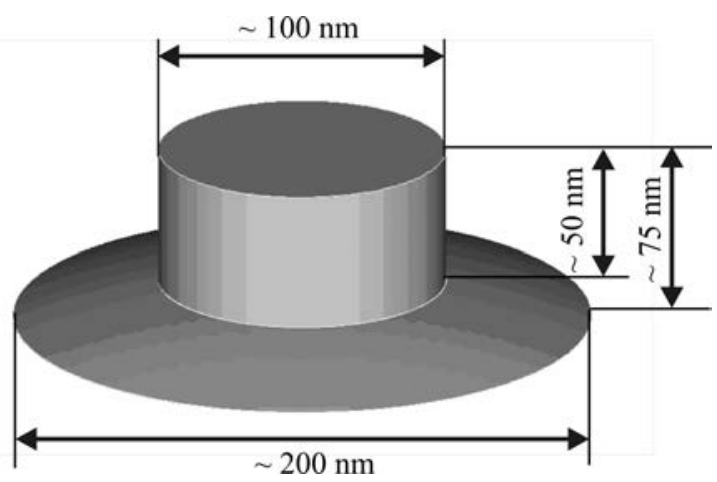

(b)

FIG. 3. The array of vertical carbon nanorods: $a-$ SEM image; $b$ - reconstruction of a single nanorod

Nanorods have a diameter of about $30 \mathrm{~nm}$ in regions with an irregular arrangement. The distance between the individual rods has a comparable value. Violation of the regularity of the registered nanorods is explained by some deviation from strict linearity, which is always present in the laser-induced periodic structures. For large-scale structures, these deviations 
are not critical, but their influence is noticeable with decreasing periodicity of the structures. Nevertheless, the tendency to form rows is visible, even in the system of irregular nanorods.

The produced nanorods have been characterized by means of nanolaboratory Ntegra Spectra (NT-MDT, Zelenograd, Russia). The Raman spectrum for these structures is shown in Fig. 4. The spectrum was recorded under ambient conditions using a He-Ne $(632.8 \mathrm{~nm})$ laser source. The strong G-peak $\left(1581 \mathrm{~cm}^{-1}\right)$ corresponds to small crystals of graphite. This significantly exceeds the dispersive D-peak $\left(1344 \mathrm{~cm}^{-1}\right)$, which is dominant for glassy carbon under ambient conditions [8]. Thus, registered nanorods may be characterized as vertical graphite crystals with a small amount of lattice defects. The broadening of the Raman peaks is a consequence of the effect of the numerous edges in arrays of nanorods [9]. The weak 2D-band is shifted to $2700 \mathrm{~cm}^{-1}$. This is typical for small-scale but the multilayer carbon structures [10].

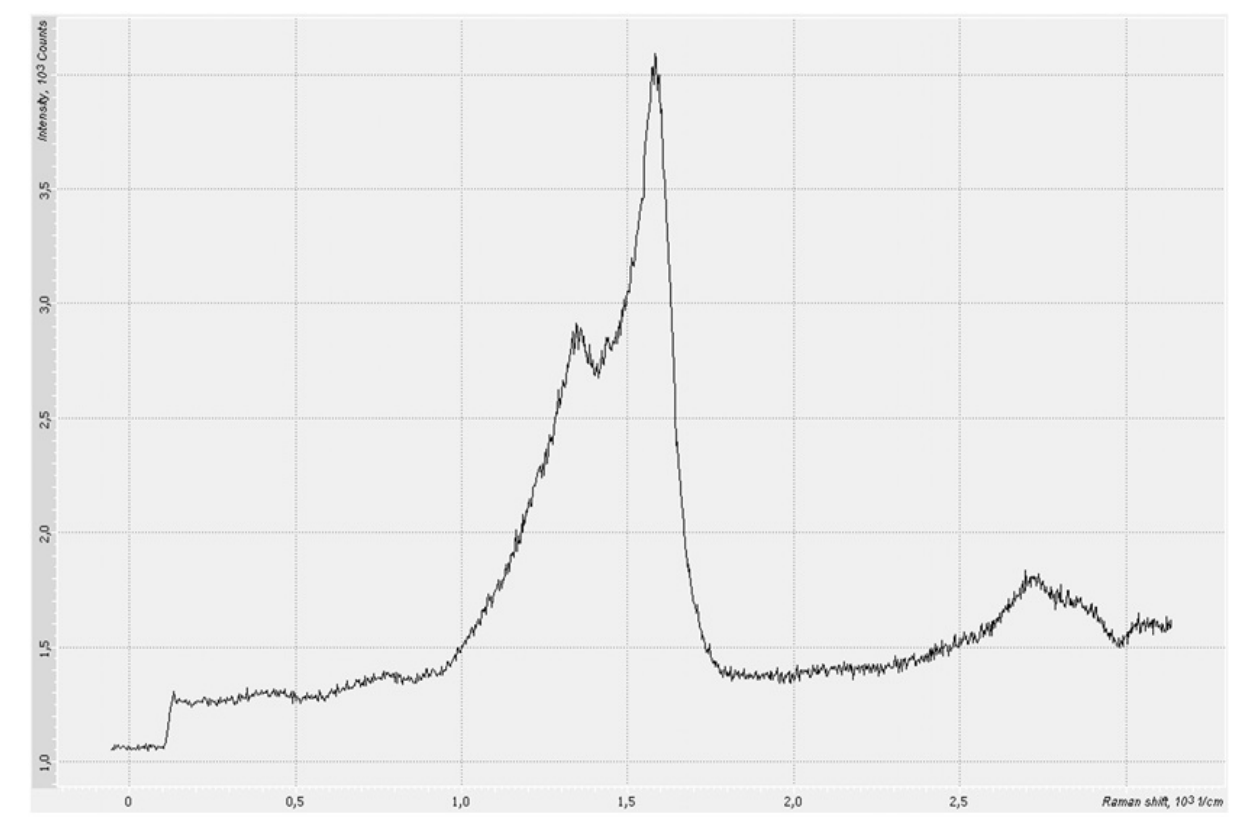

FIG. 4. Raman spectrum for array of nanorods

\section{Conclusions}

Experiments on the laser-induced liquid-phase exfoliation of graphite have demonstrated the possibility for effective application of femtosecond laser radiation pulses to produce graphene. The proposed approach not only allows short laser processing times, but also, in principle, the exclusion of a purification step for the obtained graphene, which is necessary for other methods which use additional chemical substances in their synthetic scheme. The geometric characteristics of the obtained graphene had similar parameters to that produced using currently-available experimental and commercial large scale syntheses. Also, the femtosecond laser processing of graphite in cryogenic liquids allows the formation of vertical carbon nanorod arrays on its surface.

\section{Acknowledgements}

This study was performed as part of the state task VISU 2014/13 to perform state works in the field of scientific studies and also supported under grant of the President of the Russian Federation for state support of the leading scientific schools of the RF NSh-89.2014.2 and grant of the Russian Foundation for Basic Research 14-02-97508. 


\section{References}

[1] Eletskii A.V., Iskandarova I.M., Knizhnik A.A., Krasikov D.N. Grahene: fabrication methods and thermophysical properties. Phys. Usp., 2011, 54, P. 227-258.

[2] Novoselov K.S., Jiang D., Schedin F., Booth T.J., Khotkevich V.V., Morozov S.V., Geim A.K. Two dimensional atomic crystals. PNAS, 2005, 102(30), P. 10451-10453.

[3] Makin V.S., Makin R.S. Nonlinear Interaction of Linearly Polarized Laser Radiation with Condensed Media and Overcoming the Diffraction Limit. Optics and Spectroscopy, 2012, 112(2), P. 162-167.

[4] Chu J., Peng X., Aldalbahi A., Panhuis M., Velazquez R., Feng P.X. A Simple Route to Carbon Micro- and Nanorods Hybrid Structure by Physical Vapor Deposition. J. Phys. D: Appl. Phys., 2012, 45(39), P. 395102.

[5] Mortazavi S.Z., Parvin P., Reyhani A. Fabrication of graphene based on Q-switched Nd:YAG laser ablation of graphite target in liquid nitrogen. Laser Phys. Rev. Lett., 2012, 9(7), P. 547-552.

[6] Abramov D.V., Arakelian S.M., Makov S.A., Prokoshev V.G., Khorkov K.S. Formation of a System of Microcraters on a Titanium Surface by Femtosecond Laser Radiation under Rapid Cooling Conditions. Techn. Phys. Lett., 2013, 39(8), P. 719-722.

[7] Akhmanov S.A., Emel'yanov V.I., Koroteev N.I., Seminogov V.N. Interaction of powerful laser radiation with the surfaces of semiconductors and metals: nonlinear optical effects and nonlinear optical diagnostics. Sov. Phys. Usp., 1985, 28, P. 1084-1124.

[8] Solopova N.A., Dubrovinskaia N., Dubrovinsky L. Raman spectroscopy of glassy carbon up to 60 GPa. Appl. Phys. Lett., 2013, 102(12), P. 121909.

[9] Ferrari A.C. Raman spectroscopy of graphene and graphite: Disorder, electron-phonon coupling, doping and nonadiabatic effects. Solid State Communications, 2007, 143, P. 47-57.

[10] Zheng J., Liu H., Wu B., Guo Y., Wu T., Yu G., Liu Y., Zhu D. Production of high-quality carbon nanoscrolls with microwave spark assistance in liquid nitrogen. Adv. Mater., 2011, 23(21), P. 2460-2463. 